\title{
Pain and pleasure-reconstruction after female genital mutilation
}

In 2004, the French National Health Service began to subsidize surgical reconstruction to treat dyspareunia in women who had undergone genital mutilation. The operation is now also offered to those seeking to improve their sex lives and physical appearance. In a new report, Pierre Foldés et al. describe their 11-year (1998-2009) experience of genital reconstructive surgery, finding that ,in most women, pain can be reduced and orgasm at least partially restored.

Almost 3,000 women were included in the study, the majority of whom presented with WHO type II genital mutilation (partial or total removal of the clitoris and removal of the inner and/or outer labia). Half these women experienced no pleasure during sex and at least $30 \%$ experienced some level of dyspareunia. The surgery performed was essentially the same for each patient: the clitoral stump was revealed, scar tissue resected and the glans repositioned.
Determining how these women fared 1 year after treatment was hampered because only $29 \%$ of the original cohort attended follow up. Of these women, 70\% had a visible glans and $51 \%$ were able to reach orgasm. Additionally, most reported either a reduction in or no worsening of pain, which bodes well for the future of this surgical procedure.

Studies of female genital mutilation are hampered by the lack of validated sexual function, pleasure and pain scores. Even though surgical reconstruction is feasible and seemingly successful, additional characterization of the benefits is needed. Furthermore, contributions to outcomes from social and psychological factors must also be identified, studied and addressed.

Mina Razzak

Original article Foldés, P. et al. Reconstructive surgery after female genital mutilation: a prospective cohort study. Lancet 380, 134-141 (2012) 